\title{
Influence of Distinct Radiotherapy Techniques to Induce Second Cancer Risks in Left Breast Cancer
}

\author{
Raghda A. Elgendy' ${ }^{1}$ Wahib M. Attia², Ehab M. Attalla3 ${ }^{3}$ Mostafa A. Elnaggar ${ }^{1,4}$ \\ ${ }^{1}$ Medical Physics Department, Ayady Al Mostakbal Oncology Center, Alexandria, Egypt \\ ${ }^{2}$ Physics Department, Faculty of Science, Suez Canal University, Ismailia, Egypt \\ ${ }^{3}$ Radiotherapy and Nuclear Medicine Department, National Cancer Institute, Cairo University, Giza, Egypt \\ ${ }^{4}$ Cancer Management and Research Department, Medical Research Institute, Alexandria University, Alexandria, Egypt \\ Email: raghdaelgendy_197@yahoo.com
}

How to cite this paper: Elgendy, R.A., Attia, W.M., Attalla, E.M. and Elnaggar, M.A. (2018) Influence of Distinct Radiotherapy Techniques to Induce Second Cancer Risks in Left Breast Cancer. International Journal of Medical Physics, Clinical Engineering and Radiation Oncology, 7, 193-202.

https://doi.org/10.4236/ijmpcero.2018.72017

Received: April 18, 2018

Accepted: May 25, 2018

Published: May 28, 2018

Copyright (c) 2018 by authors and Scientific Research Publishing Inc. This work is licensed under the Creative Commons Attribution International License (CC BY 4.0).

http://creativecommons.org/licenses/by/4.0/

\section{c) (i) Open Access}

\begin{abstract}
Influence of distinct radiotherapy techniques to induce second cancer risks in left breast cancer. Material and methods: Ten female patients with intact left breast cancer. Two treatment plans for each patient: 1) two tangential beams 3D-Conformal radiotherapy, 2) intensity-modulated radiotherapy (IMRT) plan. Estimation of second cancer risk from Organ equivalent dose models (OEDs) in three dose-response model for organs at risk in left breast cases. Results: The P-value for OED models in 3D-IMRT for left lung, both lung, heart, right lung, right breast, thyroid, liver and spinal cord in linear dose model was $0.016,0.005,0.019,3.95 \mathrm{E}-06,5.79 \mathrm{E}-07,0.003,1.78 \mathrm{E}-10$ and 0.000206475 respectively, for linear exponential dose model $0.0577,0.024$, $0.031,3.40 \mathrm{E}-06,3.28 \mathrm{E}-07,0.003,2.01 \mathrm{E}-10$ and 0.000120072 respectively and in plateau dose model $0.088,0.042,0.039,3.18 \mathrm{E}-06,2.53 \mathrm{E}-07,0.003$, $2.27 \mathrm{E}-10$ and $9.00535 \mathrm{E}-05$ respectively. Conclusion: organ equivalent dose models for organs at risk increasing in IMRT than tangential beams.
\end{abstract}

\section{Keywords}

Breast Cancer, Second Cancer Risks, 3DCRT, 7 Field IMRT

\section{Introduction}

The most common cancer in US women patients is breast cancer, which is the second reason for death in cancer patients. Statistics evaluate that eighth of women of America can get breast cancer [1].

Mastectomy is the traditional therapy for breast. Where nearly $1.7 / 3$ of wom- 
en have grade (I or II) breast subject to conservation breast surgery, 1.1/3 make mastectomy. Late-stage of breast cancer can develop BCS with $1 / 3 \%$, and $2 / 3 \%$ subject to mastectomy [2].

Last studies approved that mastectomy can be a cause of disturbances like depression and anxiety in addition to physical problems as breastfeed deficiency, loss of sensation in skin of chest and Deformation of body shape [3] [4].

Secondary cancer risk determined as aspect of impact about accidental and occupational irradiation then become well recognized for the age and as impact after radiotherapy. From preceding records regarding second radiation-induced cancer it confirmed occurrence over sarcoma of last irradiated area [5] [6] [7] [8] [9].

The modern studies reported dose-impact relationship for second radiation-induced cancers. They estimated that second cancer risk rises with increase dose [10]. The dose-impact relation is not linear in most tumors for doses greater than $3 \mathrm{~Gy}$. Connection for the dose rate is too significant, with less carcinogenic impact in low doses [8] [10].

The dose-response relationship is based on Radiobiological modeling to calculate harm of inducing cancer after radiotherapy. The risk rises by low doses of radiation reaching a highest rate and then reduces with the rising dose. The best way to minimize the risk of second tumors is to decrease radiation dose to sites where the dose is already low [11] [12].

From principle of organ equivalent dose to report SCR, organ mean dose is not a linear function (due to inhomogeneity of dose within tissues), the principle of OED is the response of mean weighted dose overall volume of the organ. The risk percent for different plans is synonymous to OED ratios [12] [13].

Literature showed that inversed planning (IMRT) results in better dose distribution than conformal radiotherapy (3D-CRT) for treatment of breast after conservative breast surgery [14].

\section{Materials and Methods}

1) Patient data and contouring

Ten female patients of age above 45 years old with intact left breast cancer Stage (T1-T2 and N0) and no distant metastasis. Radiotherapy is indicated after conservative surgery and after end of prescribed course of chemo if indicated, ECOG Performance Score for patients was 0 - 1. Each patient planned with twice. Each patient made CT scan with $2 \mathrm{~mm}$ slice thickness then CT images send to contouring system (Focal Pro 4.64) to delineate Target volume and organs at risk then transfer images to planning system (Computerized Medical Systems (CMS) XiO software release 4.64) where calculation made by using Artiste linear accelerator (Siemens). The two plans for each case were tangential beams 3D conformal radiation therapy and seven fields IMRT. The total dose to the target volume was 50 Gy delivered at $2 \mathrm{~Gy}$ per fraction treating daily, five days per week, the definition of target volumes and the prescribed dose accord- 
ing to recommendations of ICRU 50 and 62. Planning target volume (PTV) delineation done based on Breast Cancer Atlas (RTOG).

2) Tangential beams Three-dimensional conformal radiation therapy planning created with two tangent fields with energy $6 \mathrm{MV}$ or $6 \mathrm{MV}$ and $10 \mathrm{MV}$ depending on the patient separation. Fields were shaped at the beam's eye view to encompass the PTV shape using multi-leaf collimator (MLC).

3) Intensity-modulated radiation therapy planning created using $6 \mathrm{MV}$ with seven fields started with medial tangential beam and ended with the lateral tangent but not opposing to medial tangent field increasing angle with 5 degree and other beams distributed and divide around the target with equal space.

4) Treatment planning evaluation

Estimation of risk of secondary cancer for organs at risk by obtaining mean absorbed dose from Dose-volume histogram and calculating Organ Equivalent Dose models (OED) which is depending on the curve of dose-response to stimulate tumors and may be linear response or plateau response or linear exponential response dose.

a) Linear response to dose

$$
\text { Organ equivalent dose }=(1 / V) \sum V_{i} D_{i}
$$

b) Linear exponential response to dose

$$
\text { Organ equivalent }=(1 / V) \sum\left[\left(V_{i} D_{i}\right) e\left(-\alpha D_{i}\right)\right]
$$

c) Plateau response to dose model

$$
\text { Organ equivalent }=(1 / V) \sum V_{i}\left[\left(1-e\left(-\delta D_{i}\right)\right) / \delta\right]
$$

$V$ is the volume of all the body, $V_{i}$ is volume of organ at risk volume, and $D_{i}$ is mean dose absorbed for organ at risk, $\alpha$ and $\delta$ are a parameter to estimate response curve of organ to dose [15].

It is a dosimetric study so there is no follow up taken to check difference between two plans.

\section{Results and Discussion}

Organ Equivalent dose calculation for ten patients of intact post-lumpectomy left breast cancer with tangent beams 3DCRT and 7 fields -IMRT plans for left lung, both lung, heart, right lung, right breast, thyroid, liver and spinal cord.

\subsection{Absorbed Dose (Gy)}

From Figure 1 we see the mean dose in Gy and the SD for tangent beam 3D-CRT for left lung, both lung, heart, right lung, right breast, thyroid, liver and spinal cord for 3D-CRT were (6.777 \pm 2.612$)$, (4.243 \pm 1.847$)$, (3.501 \pm 1.494$)$, (0.173 \pm 0.023$),(0.109 \pm 0.034),(0.293 \pm 0.057),(0.353 \pm 0.132)$ and $(0.188 \pm$ $0.051)$ respectively and For IMRT, the doses were (10.496 \pm 1.684$),(8.479 \pm$ 3.196), (6.482 \pm 2.554$),(2.33 \pm 0.525),(1.619 \pm 0.642),(0.574 \pm 0.185),(2.817 \pm$ $0.343)$ and $(2.43 \pm 1.294)$ respectively. 
The mean doses of the left lung, both lung, heart, right lung, right breast, thyroid, liver and spinal cord were found to be significantly lower in tangent beams 3DCRT than IMRT plans with P value 0.00135 for left lung mean dose, 0.00191 for both lung mean dose, 0.00513 for heart mean dose, 0.000000000143 for right lung mean dose, 0.000000696 for right breast mean dose, 0.00023 for thyroid mean dose, P 0.0000000000000365 for liver mean dose and P 0.0000336 for spinal cord mean dose.

\subsection{Organ Equivalent Dose}

Figures 2-9 represent Organ Equivalent Dose models for left lung, both lung,

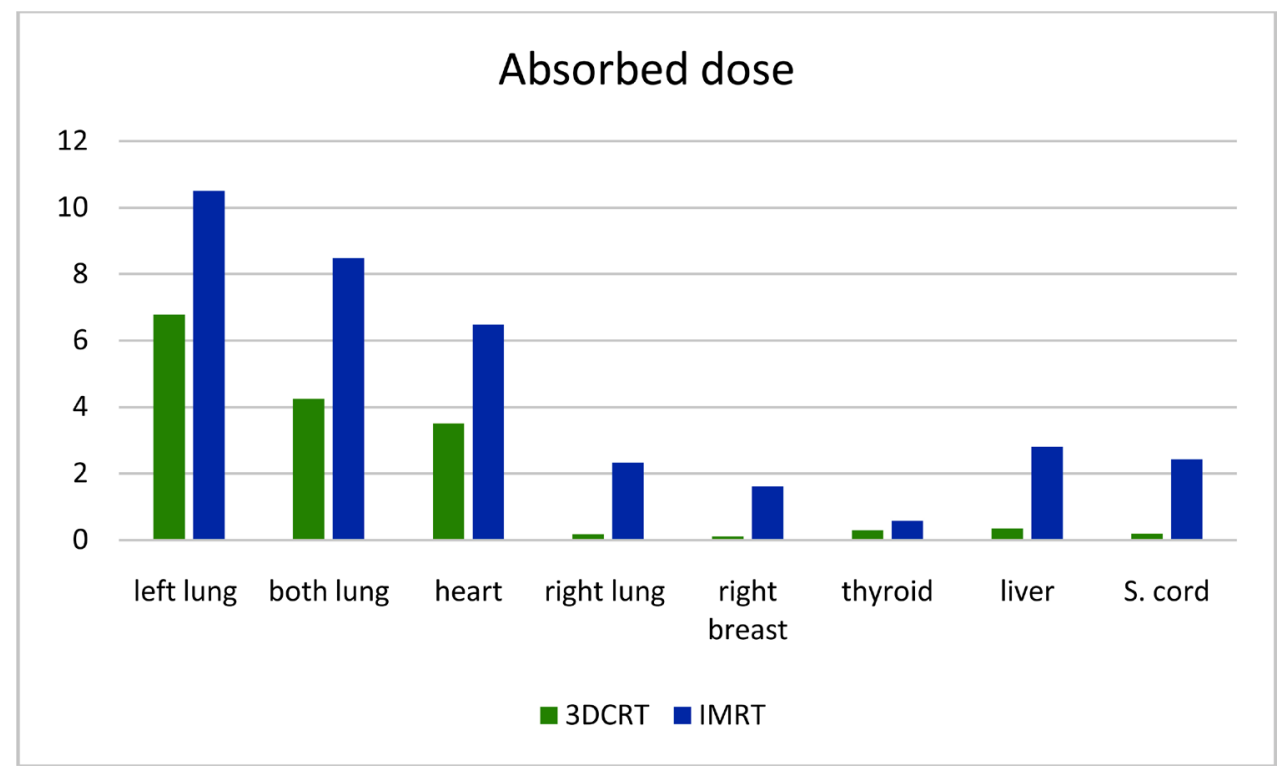

Figure 1. Average absorbed doses in various OARs for 10 left breast cancer patients treated using tangent beam 3D-CRT and IMRT.

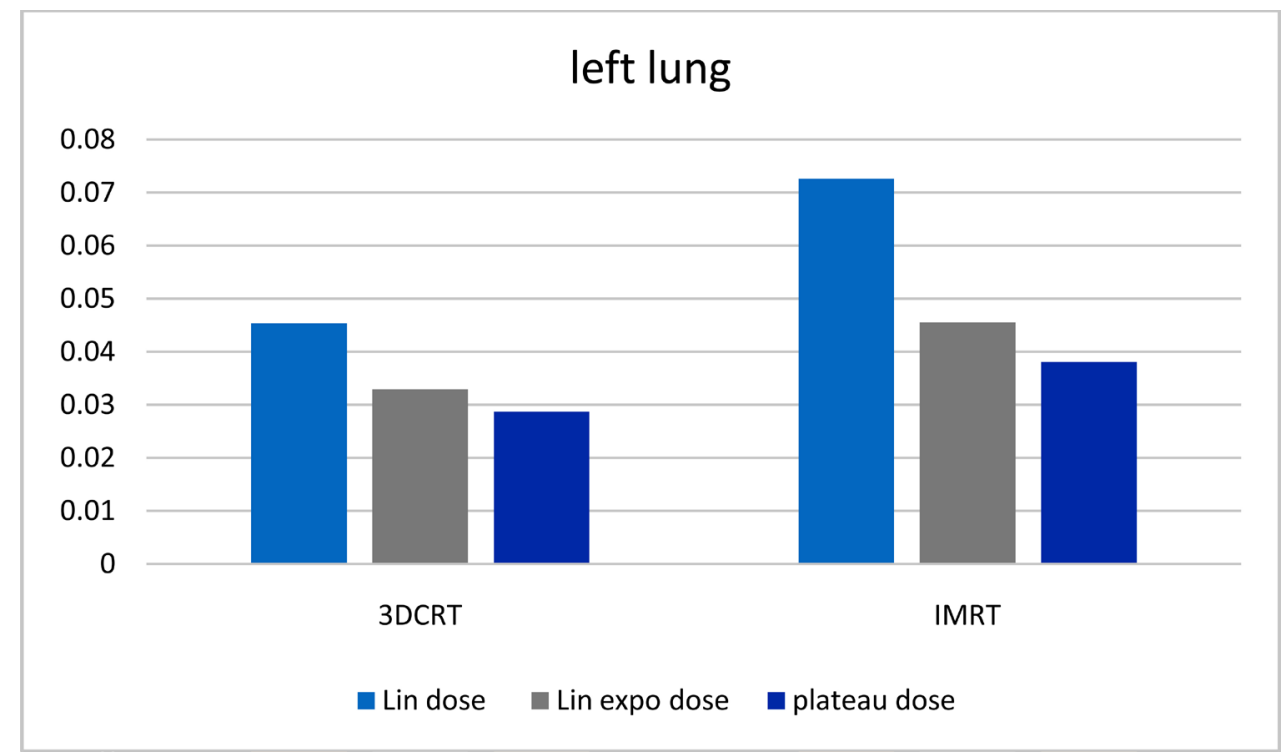

Figure 2. Organ equivalent dose for left lung in tangent beam 3D-CRT and IMRT. 


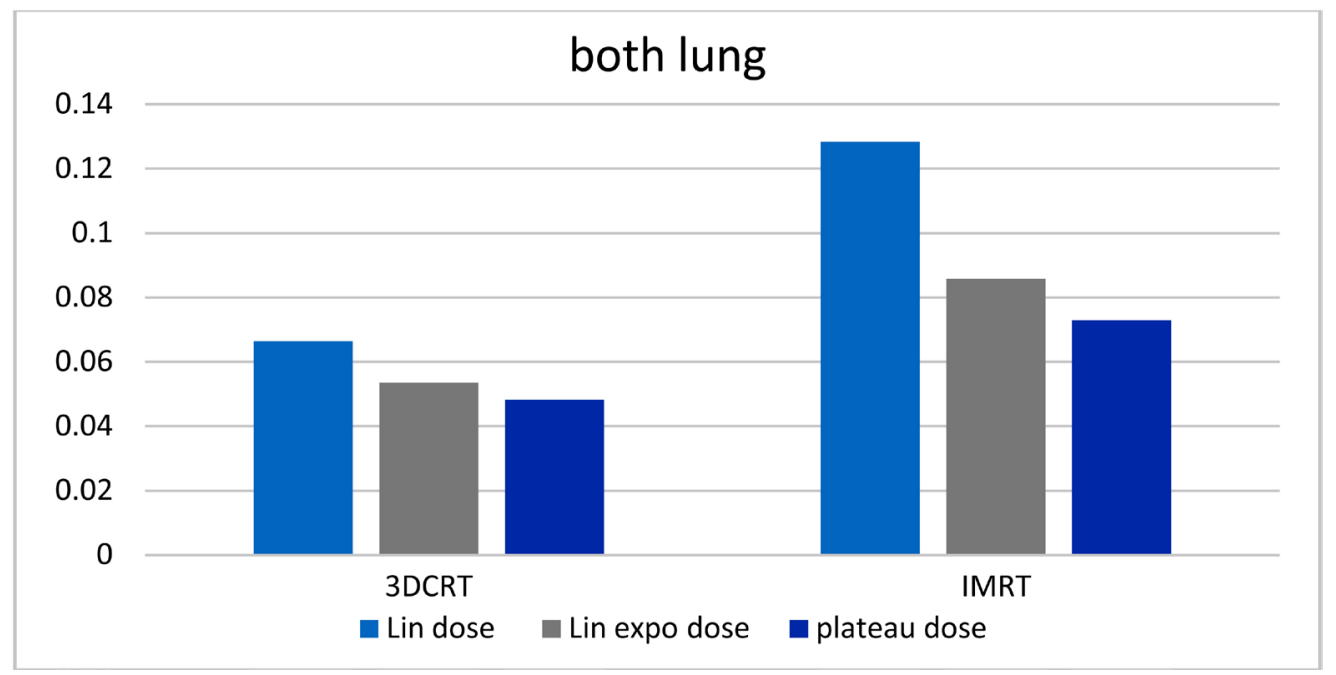

Figure 3. Organ equivalent dose for both lung in tangent beam 3D-CRT and IMRT.

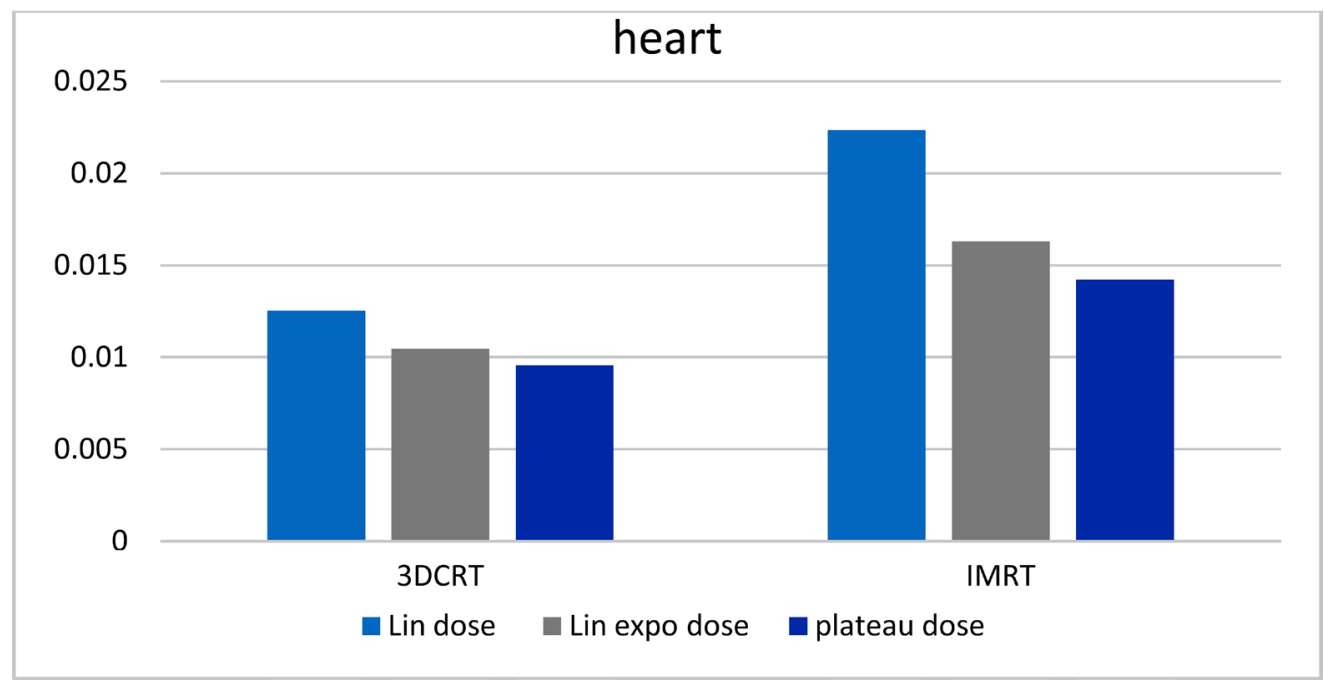

Figure 4. Organ equivalent dose for heart in tangent beam 3D-CRT and IMRT.

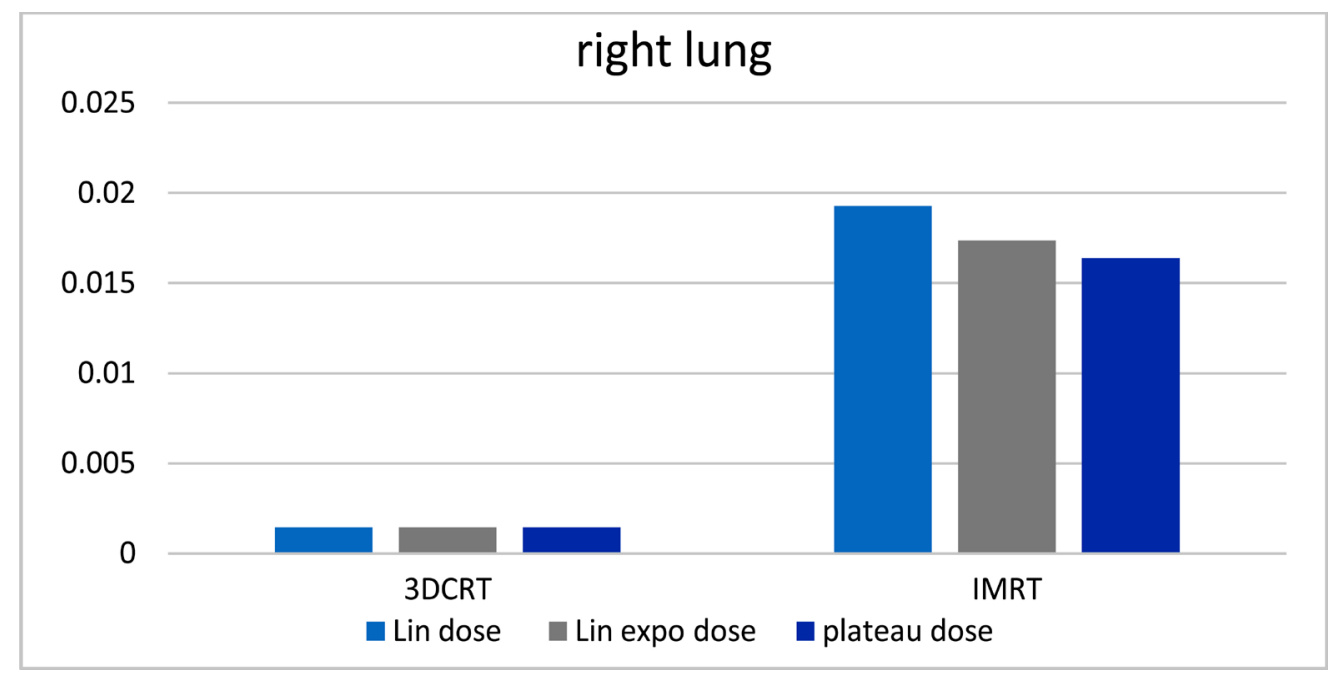

Figure 5. Organ equivalent dose for right lung in tangent beam 3D-CRT and IMRT. 


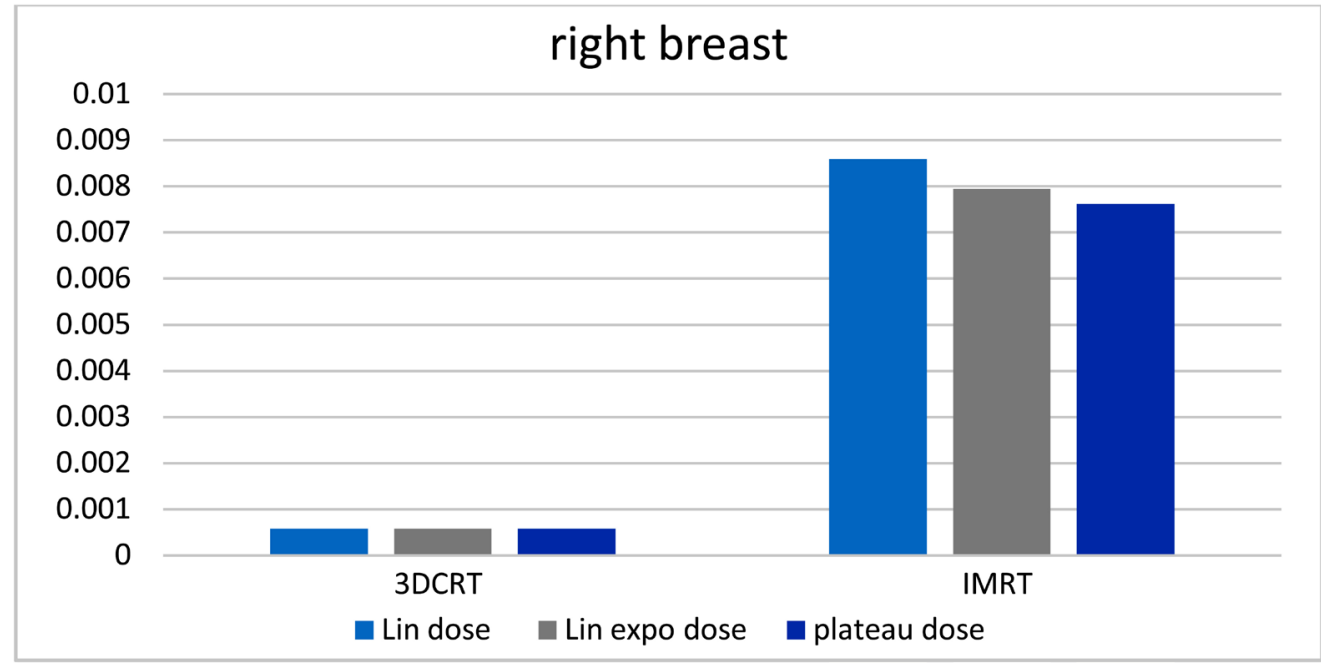

Figure 6. Organ equivalent dose for right breast in tangent beam 3D-CRT and IMRT.

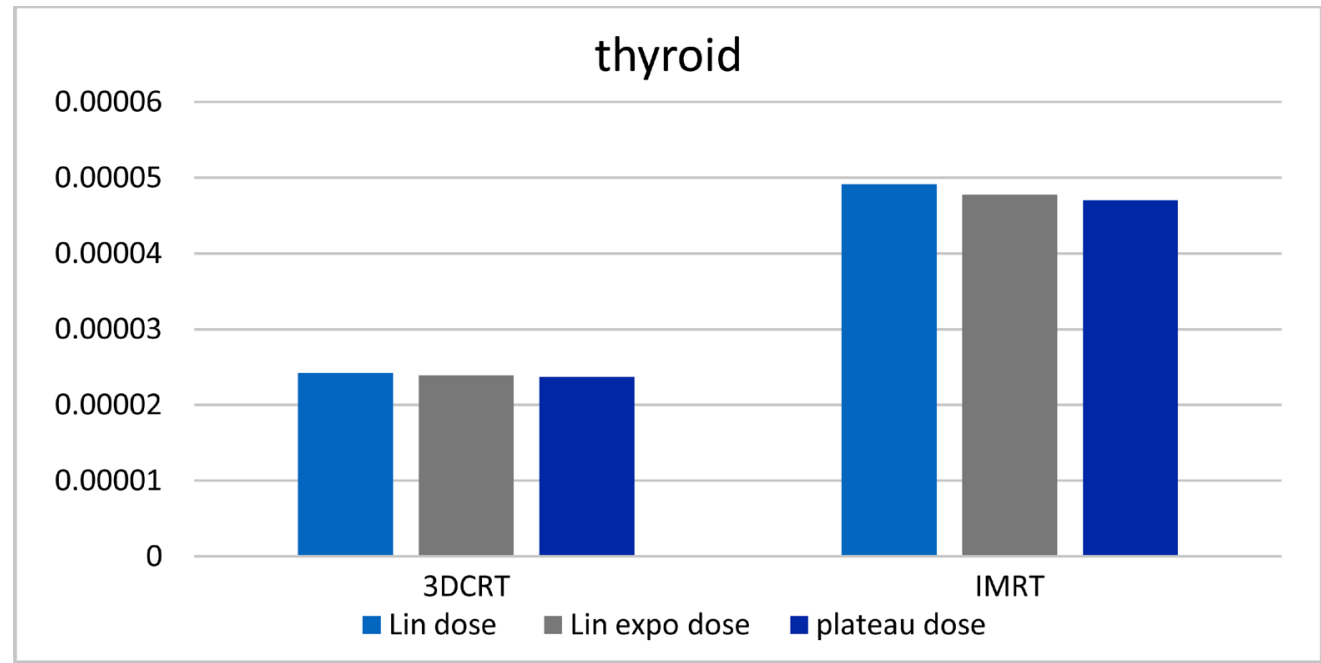

Figure 7. Organ equivalent dose for thyroid in tangent beam 3D-CRT and IMRT.

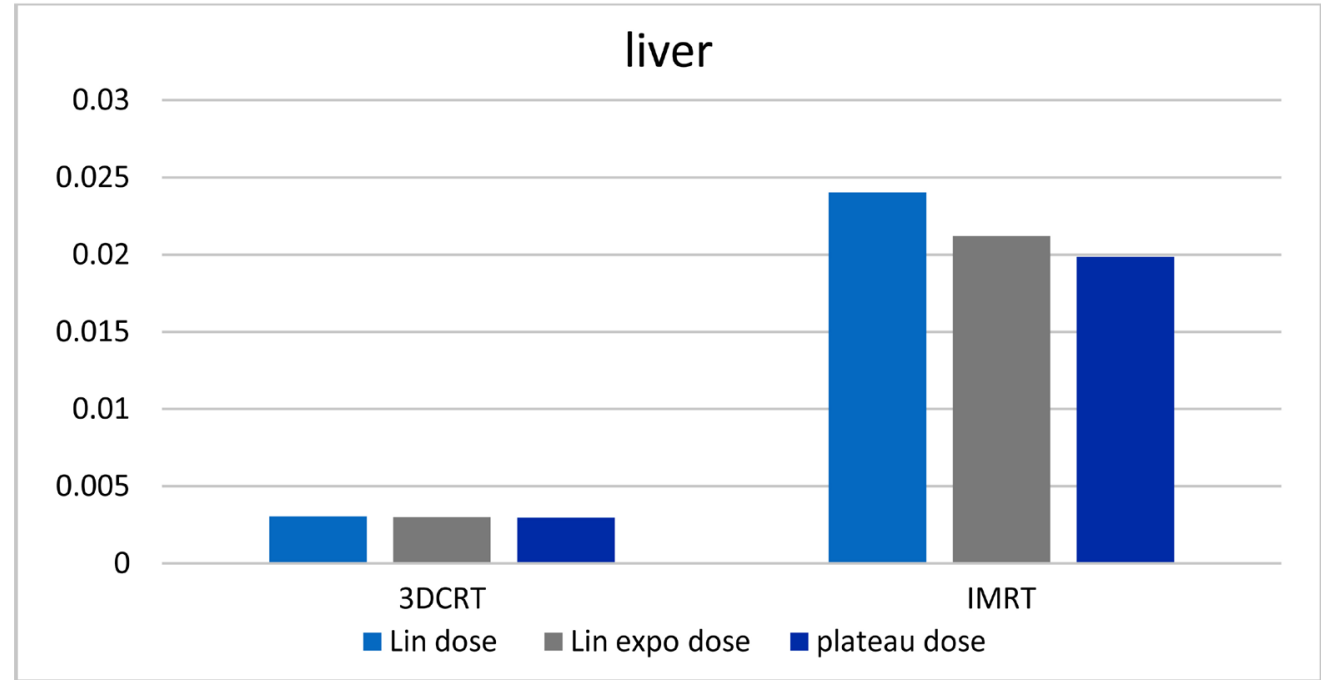

Figure 8. Organ equivalent dose for liver in tangent beam 3D-CRT and IMRT. 


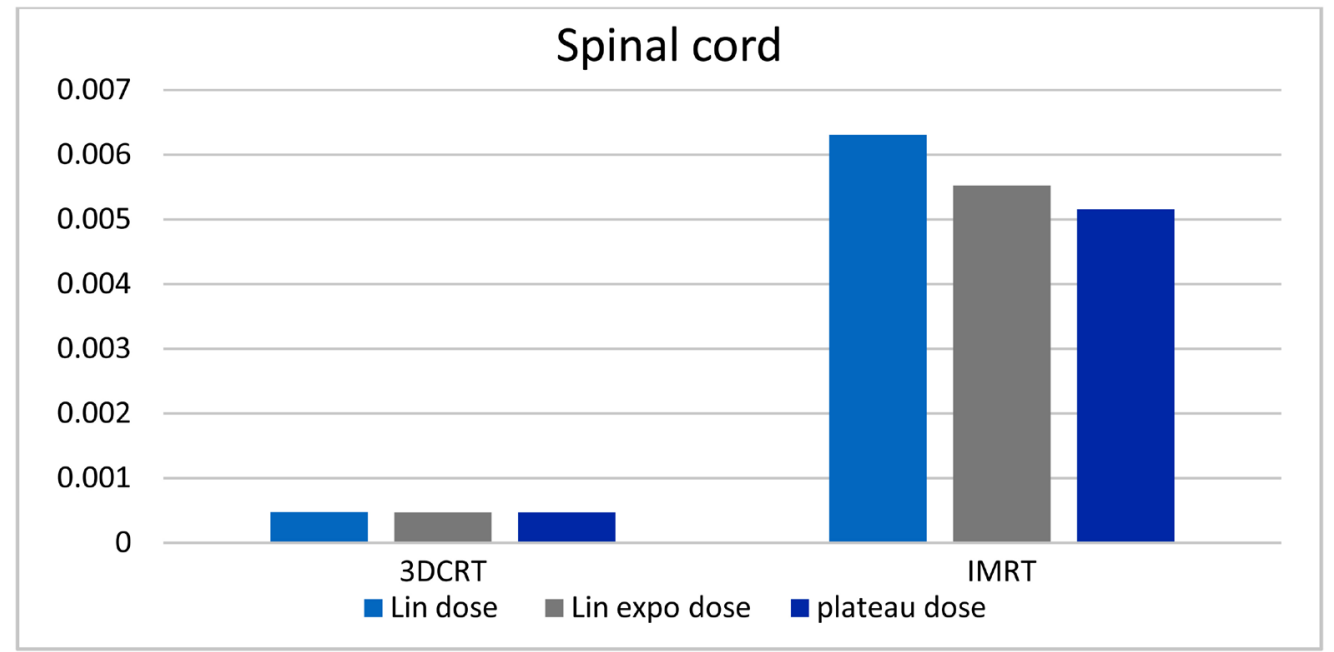

Figure 9. Organ equivalent dose for Spinal cord in tangent beam 3D-CRT and IMRT.

Table 1. P-value for OED models for second cancer risk in two tangent 3D-CRT and IMRT.

\begin{tabular}{cccc}
\hline $\begin{array}{c}\text { OAR/ } \\
\text { P-value OED }\end{array}$ & $\begin{array}{c}\text { Lin dose } \\
\text { 3D-IMRT }\end{array}$ & $\begin{array}{c}\text { Lin expo dose } \\
\text { 3D-IMRT }\end{array}$ & $\begin{array}{c}\text { Plateau dose } \\
\text { 3D-IMRT }\end{array}$ \\
\hline Left lung & 0.016 & 0.0577 & 0.088 \\
Both lung & 0.005 & 0.024 & 0.042 \\
Heart & 0.019 & 0.031 & 0.039 \\
Right lung & $3.95 \mathrm{E}-06$ & $3.40 \mathrm{E}-06$ & $3.18 \mathrm{E}-06$ \\
Right breast & $5.79 \mathrm{E}-07$ & $3.28 \mathrm{E}-07$ & $2.53 \mathrm{E}-07$ \\
Thyroid & 0.003 & 0.003 & 0.003 \\
Liver & $1.78 \mathrm{E}-10$ & $2.01 \mathrm{E}-10$ & $2.27 \mathrm{E}-10$ \\
Spinal cord & 0.000206475 & 0.000120072 & $9.00535 \mathrm{E}-05$ \\
\hline
\end{tabular}

heart, right lung, right breast, thyroid, liver and spinal cord between two tangent 3D-CRT and IMRT in ten left breast cancer.

From Figures 2-9 we notice that Organ Equivalent Dose for left lung, both lung, heart, right lung, right breast, thyroid, liver and spinal cord is lower in two tangent 3D-CRT than IMRT field.

From Table 1 we conclude that there is no significant difference for left lung in model of exponential response and plateau response between tangent 3D-CRT and IMRT but for left lung in linear dose response model all other organs at risk there is significant difference for organ equivalent dose models in two tangent 3D-CRT and IMRT, where intensity modulated radiation therapy can induce second cancer risk for organ at risk than tangent beams three dimensional conformal radiation therapy.

The secondary of the dose depended on the distance from the isocenter and their modalities. Where TOMO, is less than or compatible with the secondary of the dose and OED from conventional IMRT and VMAT but TOMO is similar to 
IMRT and VMAT as increasing the distance from the field edge [16].

Older radiation techniques increase the secondary risk where more volumes take high doses [17] [18] [19] [20] [21]. Nowadays radiotherapy techniques decrease risk of second cancer by reducing the organ at risk dose [14]. Smoke is significantly increase risk of lung cancer in patients even using modern radiation techniques [22] [23]. The risk of second cancers increases by using multi-field IMRT when comparing with 3D-CRT where enormous normal tissue exposed to lower doses [14] [24].

Organs near to the PTV have rising risk to stimulate second cancer [25]. Second cancer risks for in-field organs were more than for organs outside of treatment field with an exception for ITF and VMAT plan [16]. Optimization and conformation to treated volume consequence reduce in relative OED in all three relationships dose-response. Volumetric arc therapy and intensity modulated radiation therapy plan decreases volume receive high dose close to normal organs by increasing the numbers of field to attain more conformed dose while increasing spare of the low dose across a larger volume while a resulting a high doses to skin [12].

Treatment modality used in left breast cancer may be a relative cause of second cancer risk [26]. Where doses for organs at a distant from the treated volume in volumetric modulated arc therapy increases the risk of second cancer to these organs. Conventional plans compared to new complicated plans are reported to increase second cancer risk organs at risk like in heart and left lung [12].

Radiotherapy patients sometimes have increasing risk to develop second cancer due to some factors as genetic, environment and their culture which is more dominant than risk from radiation [15] from dose-response curve we can't know which model is reasonable for carcinogenesis but Plateau model may be suitable to evaluate risk of second cancer [12].

\section{Conclusions}

From the present study we conclude that the mean doses of the left lung, both lung, heart, right lung, right breast, thyroid and liver were found to be significantly higher in intensity modulated radiation than tangent beams three dimensional conformal radiation therapy plans for left breast cancer.

The second cancer risk for organ equivalent dose models for organs at risk increasing in intensity modulated radiation than tangent beams three dimensional conformal radiation therapy.

\section{Acknowledgements}

This work was supported by Ayady Al Mostakbal Oncology Center, Alexandria, Egypt. We would like to thank Dr. Assem Rostom, MD, FRCR General Manager and Consultant Clinical Oncologist Ayadi Al-Mostakbal Charity Clinical Oncology Center for encourage and supporting all the time. 


\section{References}

[1] DeSantis, C., Ma, J., Bryan, L., et al. (2014) Breast Cancer Statistics. CA: A Cancer Journal for Clinicians, 64, 52-62. https://doi.org/10.3322/caac.21203

[2] Freedman, A.N., Yu, B., Gail, M.H., et al. (2011) Benefit/Risk Assessment for Breast Cancer Chemoprevention with Raloxifene or Tamoxifen for Women Age 50 Years or Older. Journal of Clinical Oncology, 29, 2327-2333. https://doi.org/10.1200/JCO.2010.33.0258

[3] Cordeiro, P.G. (2008) Breast Reconstruction after Surgery for Breast Cancer. The New England Journal of Medicine, 359, 1590-1601. https://doi.org/10.1056/NEJMct0802899

[4] Zhang, P., Li, C.Z., Wu, C.T., Jiao, G.M., Yan, F., Zhu, H.C. and Zhang, X.-P. (2017) Comparison of Immediate Breast Reconstruction after Mastectomy and Mastectomy Alone for Breast Cancer: A Meta-Analysis. European Journal of Surgical Oncology, 43, 285-293. https://doi.org/10.1016/j.ejso.2016.07.006

[5] Cahan, W.G., Woodard, H.Q., Higinbotham, L., Stewart, F.W. and Coley, B.L. (1948) Sarcoma Arising in Irradiated Bone: Report of Eleven Cases. Cancer, 1, 3-29. https://doi.org/10.1002/1097-0142(194805)1:1<3::AID-CNCR2820010103>3.0.CO;2-7

[6] Suit, H., Goldberg, S., Niemierko, A., et al. (2007) Secondary Carcinogenesis in Patients Treated with Radiation: A Review on radiation-Induced Cancers in Human, Non-Human Primate, Canine and Rodent Subjects. Radiation Research, 167, 12-42. https://doi.org/10.1667/RR0527.1

[7] Xu, X.G., Bernarz, B. and Paganetti, H. (2008) A Review of Dosimetry Studies on External-Beam Radiation Treatment with Respect to Second Cancer Induction. Physics in Medicine \& Biology, 53, R193-R241. https://doi.org/10.1088/0031-9155/53/13/R01

[8] Tubiana, M. (2009) Can We Reduce the Incidence of Second Primary Malignancies Occurring after Radiotherapy: A Critical Review. Radiotherapy \& Oncology, 91, 4-15. https://doi.org/10.1016/j.radonc.2008.12.016

[9] Shilkrut, M., Belkacemi, Y. and Kuten, A. (2012) Secondary Malignancies in Survivors of Breast Cancer: How to Overcome the Risk. Critical Reviews in Oncology/Hematology, 84, 86-89. https://doi.org/10.1016/j.critrevonc.2010.06.007

[10] Woo, T.C., Pignol, J.P., Rakovitch, E., et al. (2006) Body Radiation Exposure in Breast Cancer Radiotherapy: Impact of Breast IMRT and Virtual Wedge Compensation Techniques. International Journal of Radiation Oncology, Biology, Physics, 65, 52-58. https://doi.org/10.1016/j.ijrobp.2005.11.023

[11] Sachs, R.K., Brener, D.J., et al. (2005) Solid Tumor Risks after High Doses of Ionizing Radiation. Proceedings of the National Academy of Sciences of the United States of America, 102, 13040-13045. https://doi.org/10.1073/pnas.0506648102

[12] Sakthivela, V., Mania, G., Mani, S. and Boopathy, R. (2017) Comparison of Treatment Planning Techniques in Treatment of Carcinoma of Left Breast: Second Cancer Perspective. Radiation Physics and Chemistry, 132, 41-45. https://doi.org/10.1016/j.radphyschem.2016.11.009

[13] Schneider, U., Zwahlen, D., Ross, D., et al. (2005) Estimation of Radiation Induced Cancer from Three Dimensional Dose Distributions: Concept of Organ Equivalent Dose. International Journal of Radiation Oncology, Biology, Physics, 61, 1510-1515. https://doi.org/10.1016/j.ijrobp.2004.12.040

[14] Rudat, V., Alaradi, A., Mohamed, A., AI-Yahya, K. and Altuwaijri, S. (2011) Tangential Beam IMRT versus Tangential Beam 3D-CRT of the Chest Wall in Post Mastectomy Breast Cancer Patients: A Dosimetric Comparison. Radiotherapy \& 
Oncology, 6, 26. https://doi.org/10.1186/1748-717X-6-26

[15] Schneider, U., Walsh, L., et al. (2008) Cancer Risk Estimates from the Combined Japanese A-Bomb and Hodgkins Cohorts for Doses Relevant to Radiotherapy. Radiation and Environmental Biophysics, 47, 253-263. https://doi.org/10.1007/s00411-007-0151-y

[16] Kim, D., Chung, W., Shin, D., Hong, Park, S.H., Park, S.Y., Chung, K., Lim, Y.K., Shin, D., Lee, S.B., Lee, H.H. and Yoon, M. (2013) Risk of Second Cancer from Scattered Radiation of Intensity-Modulated Radiotherapies with Lung Cancer. Radiation Oncology, 8, 47. https://doi.org/10.1186/1748-717X-8-47

[17] Zablotska, L.B. and Neugut, A.I. (2003) Lung Carcinoma after Radiation Therapy in Women Treated with Lumpectomy or Mastectomy for Primary Breast Carcinoma. Cancer, 97, 1404-1411. https://doi.org/10.1002/cncr.11214

[18] Prochazka, M., Granath, F., Ekbom, A., Shields, P.G. and Hall, P. (2002) Lung Cancer risks in Women with Previous Breast Cancer. European Journal of Cancer, 38, 1520-1525. https://doi.org/10.1016/S0959-8049(02)00089-8

[19] Galper, S., Gelman, R., Recht, A., Silver, B., Kohli, A., Wong, J.S., Van Buren, T., Baldini, E.H. and Harris, J.R. (2002) Second Nonbreast Malignancies after Conservative Surgery and Radiation Therapy for Early-Stage Breast Cancer. International Journal of Radiation Oncology, Biology, Physics, 52, 406-414. https://doi.org/10.1016/S0360-3016(01)02661-X

[20] Fisher, B., Anderson, S., Bryant, J., Margolese, R.G., Deutsch, M., Fisher, E.R., Jeong, J.H. and Wolmark, N. (2002) Twenty-Year Follow-Up of a Randomized Trial Comparing Total Mastectomy, Lumpectomy, and Lumpectomy plus Irradiation for the Treatment of Invasive Breast Cancer. The New England Journal of Medicine, 347, 1233-1241. https://doi.org/10.1056/NEJMoa022152

[21] Roychoudhuri, R., Evans, H., Robinson, D. and Moller, H. (2004) Radiation-Induced Malignancies Following Radiotherapy for Breast Cancer. British Journal of Cancer, 91, 868-872. https://doi.org/10.1038/sj.bjc.6602084

[22] van Dongen, J.A., Voogd, A.C., Fentiman, I.S., Legrand, C., Sylvester, R.J., Tong, D., van der Schueren, E., Helle, P.A., van Zijl, K. and Bartelink, H. (2000) Long-Term Results of a Randomized Trial Comparing Breast-Conserving Therapy with Mastectomy: European Organization for Research and Treatment of Cancer 10801 Trial. Journal of the National Cancer Institute, 92, 1143-1150. https://doi.org/10.1093/jnci/92.14.1143

[23] Blichert-Toft, M., Nielsen, M., During, M., Moller, S., Rank, F., Overgaard, M. and Mouridsen, H.T. (2008) Long-Term Results of Breast Conserving Surgery vs. Mastectomy for Early Stage Invasive Breast Cancer: 20-Year Follow-Up of the Danish Randomized DBCG-82TM Protocol. Acta Oncologica, 47, 672-681.

[24] Hall, E.J. and Wuu, C.S. (2003) Radiation-Induced Second Cancers: The Impact of 3D-CRT and IMRT. International Journal of Radiation Oncology, Biology, Physics, 56, 83-88. https://doi.org/10.1016/S0360-3016(03)00073-7

[25] Chaturvedi, A.K., Ahamad, A.A., Iyer, R.B., et al. (2007) Feasibility of Dose Escalation Using Intensity Modulated Radiotherapy in Posthysterectomy Cervical Carcinoma. Journal of the National Cancer Institute, 99, 1634-1643. https://doi.org/10.1093/jnci/djm201

[26] Boice, J.D., Day, N.E., Anderson, A., et al. (1985) Second Cancers Following Radiation Treatment for Cervical Cancer. Journal of the National Cancer Institute, 74, 955-959. 